\title{
Interaction des facteurs héliothermiques sur la croissance de trois espèces du genre Scenedesmus
}

\author{
A. Benider ${ }^{1 *}$ \\ M. Tahiri ${ }^{1}$ \\ M. Belkoura 1 \\ A. Dauta 2
}

Mots-clés : Scenedesmus abundans, Scenedesmus dimorphus, Scenedesmus ecornis, température, intensité lumineuse, photopériode, taux de croissance, modélisation.

Dans le cadre d'un programme de sélection de microalgues potentiellement valorisables, l'influence des facteurs héliothermiques sur la croissance de trois espèces de Scenedesmus ( $S$. abundans, $S$. dimorphus et $S$. ecornis) a été étudiée sur des cultures réalisées en milieu fini et en conditions nutritionnelles non limitantes. Pour chacune des trois espèces, les effets de l'intensité lumineuse (de 30 à $560 \mu$ mole quanta. $\mathrm{m}^{-2} \cdot \mathrm{s}^{-1}$ ), de la température (de 10 à $35^{\circ} \mathrm{C}$ ) et de la photopériode sur les taux de croissance ont été mesurés puis modélisés. Les résultats obtenus démontrent le caractère mésophile des trois microalgues avec un optimum thermique de $30^{\circ} \mathrm{C}$ pour $S$. ecornis et de $35^{\circ} \mathrm{C}$ pour les deux autres espèces. L'existence d'une interaction étroite entre l'intensité lumineuse et la température sur la croissance des trois microalgues a également été mise en évidence. De même, une influence très nette de la photopériode sur la croissance des trois espèces a été relevée. Dans les conditions optimales de culture, les taux de croissance maximaux observés sont respectivement de $2,1 \mathrm{~d}^{-1}$ pour $S$. ecornis, $1,95 \mathrm{~d}^{-1}$ pour $S$. abundans et $1,5 \mathrm{~d}^{-1}$ pour $S$. dimorphus. Les effets des facteurs héliothermiques sur la croissance des microalgues étudiées ont pu être restitués de manière satisfaisante par trois modèles mathématiques. Les résultats obtenus indiquent que les trois espèces de $S c e-$ nedesmus sont parfaitement adaptées aux conditions climatiques de la région de Marrakech. Leur culture en masse, à des fins appliquées, pourrait donc être envisageable.

\section{Interacting effect of heliothermic factors on the growth rate of 3 Scenedesmus species.}

Keywords : Scenedesmus abundans, Scenedesmus dimorphus, Scenedesmus ecornis, temperature, light intensity, photoperiod, growth rate, modelling.

Within the framework of a microalgae screening program, three species of Scenedesmus (S. abundans, $S$. dimorphus and $S$. ecomis) were studied in batches and in non-nutrient limited conditions. For each species, the effects of light intensity (from 30 to $560 \mu$ mole quanta. $\mathrm{m}^{-2} . \mathrm{s}^{-1}$ ), temperature (from 10 to $35^{\circ} \mathrm{C}$ ) and daylength on the growth rate have been measured and mathematically formulated. The results obtained show that the three microalgae are mesophilic strains, with an optimal growth temperature of $30^{\circ} \mathrm{C}$ for $S$. ecornis and $35^{\circ} \mathrm{C}$ for the two other species. Interaction between temperature and light intensity on growth has been demonstrated for the three species. In the same way, a positive effect of daylength on the growth rates of Scenedesmus spp. has been recorded. Under optimal conditions, the maximum growth rates are respectively of $2,1 \mathrm{~d}^{-1}$ for $S$. ecornis, $1,95 \mathrm{~d}^{-1}$ for $S$. abundans and 1,5 $\mathrm{d}^{-1}$ for $S$. dimorphus. Moreover, the effects of heliothermic factors on the growth rate of the three species have been reproduced satisfactorily by using three mathematical models. This study indicates that the three species of Scenedesmus are adapted to the climatic conditions of the Marrakech area. So, for application purposes, the largescale culture of these species could be possible.

\footnotetext{
1. Equipe Biotechnologie Algale. Laboratoire de Physiologie Végétale, Faculté des Sciences Semlalia, Avenue Prince Moulay Abdallah, B.P. 2390, Marrakech, Maroc.

* e-mail: benider@ucam.ac.ma

2. Centre d'Ecologie des Systèmes Aquatiques Continentaux, UMR C.5576 CNRS, Université Paul Sabatier, 118 route de Narbonne, F-31062 Toulouse Cedex, France.
} 


\section{Introduction}

Au cours des cinq dernières décennies, de nombreux travaux ont été réalisés sur les microalgues avec pour principal objectif la valorisation de ces organismes dans divers domaines d'application : alimentation, médecine, biotraitement des effluents, etc... Parmi les chlorophycées exploitées actuellement, les espèces du genre Scenedesmus occupent une place prépondérante en raison de leur productivité élevée, (jusqu'à 98 tonnes de matière sèche par hectare et par an, Soeder \& Hegewald 1988) et de leur composition biochimique intéressante, (teneurs en protéines pouvant atteindre jusqu'à $50 \%$ de la matière sèche, Richmond 1986a).

Les premières tentatives de production à l'échelle pilote ont été menées au Pérou au début des années 1970 avec l'espèce Scenedesmus acutus var. alternans Nr. 276-3a (Castillo et al. 1980). Depuis, de nombreux systèmes de production en masse ont été développés dans plusieurs pays, pour diverses espèces appartenant à ce genre (Stengel \& Soeder 1975, Payer et al. 1978, Becker \& Venkataraman 1980, 1982, Dilov et al. 1985, El-Fouly et al. 1985, Heussler 1985). Les rendements de ces systèmes restent cependant variables selon les caractéristiques intrinsèques des espèces utilisées et la structure des unités de production, mais également en fonction de l'influence des facteurs environnementaux (température et éclairement) sur la croissance de ces microalgues. Ainsi, à côté de l'amélioration des techniques de culture, la recherche de souches de Scenedesmus adaptées aux conditions climatiques des sites de production est une des stratégies préconisées dans ce domaine. Dans le cadre d'un programme de sélection de microalgues à intérêt alimentaire et industriel, développé par le laboratoire de Marrakech, nous avons été amenés à évaluer les potentialités de valorisation de trois espèces du genre Scenedesmus ( $S$. abundans, $S$. dimorphus et $S$. ecormis) isolées à partir de deux écosystèmes aquatiques marocains. Parallèlement aux analyses de leur composition biochimique (Tahiri et al. 2000, Benider, 2001), un des principaux objectifs de ce programme est de déterminer leurs caractéristiques écophysiologiques en relation avec les facteurs héliothermiques. Le travail présenté a donc pour but d'étudier les interactions entre la lumière et la température sur le taux de croissance des trois microalgues. Il s'attache également à fournir un ensemble de données permettant de développer ultérieurement un modèle intégré de simulation de la croissance de ces espèces susceptible d'être utilisé pour prédire, à moyen terme, la productivité des systèmes de culture en masse.

\section{Matériel et méthodes}

Les algues vertes Scenedesmus abundans (Kirchner) Chod. et Scenedesmus ecornis (Ehrenb.) Chod. ont été isolées du lac de barrage Lalla Takerkoust (région de Marrakech, Maroc). L'espèce Scenedesmus dimorphus (Turp.) Kütz a été isolée du canal algal à haut rendement (CAHR) de l'Institut Agronomique et Vétérinaire Hassan II (Rabat, Maroc). Les différentes souches d'algues utilisées dans cette étude sont monoalgales mais non axéniques. Cependant, l'utilisation d'un milieu de culture entièrement minéral (Dauta 1982) et la manipulation en conditions d'asepsie stricte réduisent les risques de contamination bactérienne. Les souches mères sont cultivées en milieu fini (batch). Elles sont maintenues en phase exponentielle de croissance par des repiquages fréquents ( 2 à 3 jours), dans des conditions de température et d'intensité lumineuse sub-optimales (i.e. $25^{\circ} \mathrm{C}, 250 \mu$ mole quanta. $\mathrm{m}^{-2} \cdot \mathrm{s}^{-1}$ ). Afin d'éviter la sédimentation cellulaire et de prévenir 1 'appauvrissement du milieu de culture en $\mathrm{CO}_{2}$, les cultures sont fortement bullées $\left(0,51 \mathrm{~d}^{\prime}\right.$ air. $\mathrm{l}^{-1}$ culture. $\mathrm{min}^{-1}$ ) avec de l'air comprimé filtré au travers d'une capsule de $0,2 \mu \mathrm{m}$ de porosité (Gelman). Les expériences sont réalisées dans une enceinte thermorégulée, sous une photopériode de 15 heures de lumière pour 9 heures d'obscurité $(15 / 9 ; \mathrm{L} / \mathrm{O})$. La lumière est apportée par une lampe Phytoclaude de 400 watts placée à la verticale des cultures. L'intensité lumineuse incidente est mesurée à l'aide d'un quantamètre $\mathrm{Li}$-Cor 185A.

Avant les expériences destinées à la mesure des taux de croissance, les cultures sont placées pendant 24 heures à l'obscurité (synchronisation et non préadaptation à la lumière). Au terme de cette phase de synchronisation, les cultures sont largement diluées dans un milieu neuf afin d'éviter tout phénomène d'auto-ombrage (densité de population de la culture $=3.10^{5} \mathrm{cel}$ lules. $\mathrm{ml}^{-1}$ ), puis réparties dans une série d'erlenmeyers de $250 \mathrm{ml}$. Pour chacune des six températures testées (entre 10 et $35^{\circ} \mathrm{C}$ ), les erlenmeyers sont exposés à des intensités lumineuses comprises entre 30 et $560 \mu$ mole quanta. $\mathrm{m}^{-2} \cdot \mathrm{s}^{-1}$, sous une photopériode de $15 / 9(\mathrm{~L} / \mathrm{O})$. Les cultures destinées à l'étude de l'influence de la photopériode sur le taux de croissance sont incubées à $30^{\circ} \mathrm{C}$, sous une intensité lumineuse de $350 \mu$ mole quanta. $\mathrm{m}^{-2} \cdot \mathrm{s}^{-1}$ et à différentes photopériodes $(3 / 21$, $6 / 18, \ldots, 24 / 0, \mathrm{~L} / \mathrm{O})$.

Les taux de croissance sont déterminés à partir des mesures d'absorbance à $750 \mathrm{~nm}$ (DO ${ }^{750}$, cuve de $10 \mathrm{~mm}$, spectrophotomètre Secomam S750). Des expériences préliminaires ont montré en effet l'existence, pour les trois espèces d'algues, d'une forte corrélation 


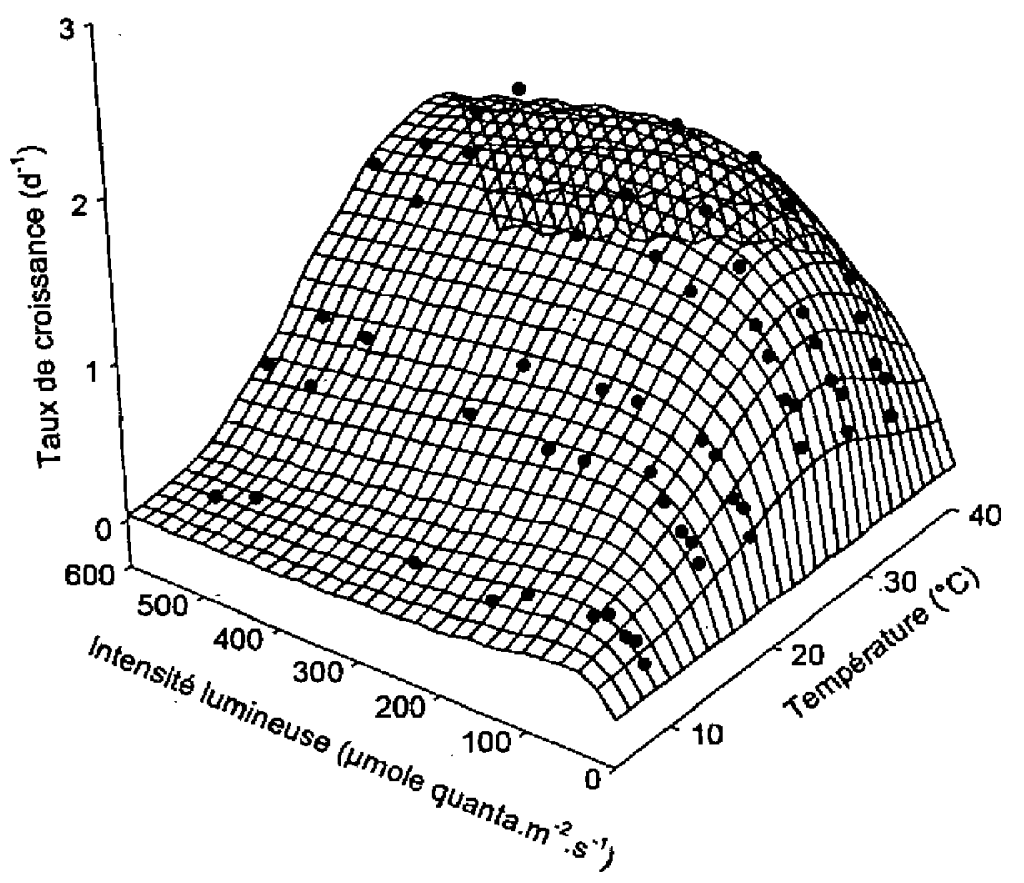

Fig. 1. Interaction de l'intensité lumineuse et de la température sur le taux de croissance de Scenedesmus abundans.

Fig. 1. Interaction between light intensity and temperature on the growth rate of Scenedesmus abundans.

positive $(r=0,98)$ entre la densité de la population de cellules et la $\mathrm{DO}_{750}$. Les mesures sont effectuées au début du cycle lumineux $\left(A_{0}\right)$ et après 24 heures d'incubation $\left(A_{1}\right)$. Les taux de croissance sont calculés selon la relation :

$\mu=\operatorname{Ln}\left(A_{1} / A_{0}\right)$, pour un intervalle de temps de 1 jour.

\section{Résultats}

Les figures 1, 2 et 3 illustrent l'évolution du taux de croissance des trois espèces de Scenedesmus en fonction des facteurs héliothermiques (intensité lumineuse et température). Pour chacune des températures testées, l'utilisation du modèle de Peeters \& Eilers (1978) permet d'ajuster de manière très satisfaisante les données expérimentales enregistrées (Figs. 1, 2, 3). En effet, ce modèle (équation 1) reproduit parfaitement l'accroissement du taux de croissance avec l'intensité lumineuse (I) jusqu à une valeur maximale ( $\mu$ max) correspondant à un éclairement optimal (Iopt). Il restitue également le phénomène de photoinhibition de la croissance aux fortes intensités lumineuses (lorsque I $>$ Iopt).

$\mu=\mu \max .2$. $(1+\beta) .(\mathrm{I} / \mathrm{Iopt}) /\left[(\mathrm{I} / \mathrm{Iopt})^{2}+2 . \mathrm{I} / \mathrm{Iopt} . \beta\right.$ $+1]$ (1)

où $\mu$ représente le taux de croissance pour une intensité lumineuse I, $\mu$ max et Iopt correspondent respectivement au taux de croissance maximal et à l'intensité lumineuse pour laquelle $\mu=\mu \max , \beta$ est un facteur permettant de prendre en compte le phénomène de photoinhibition aux fortes intensités lumineuses.

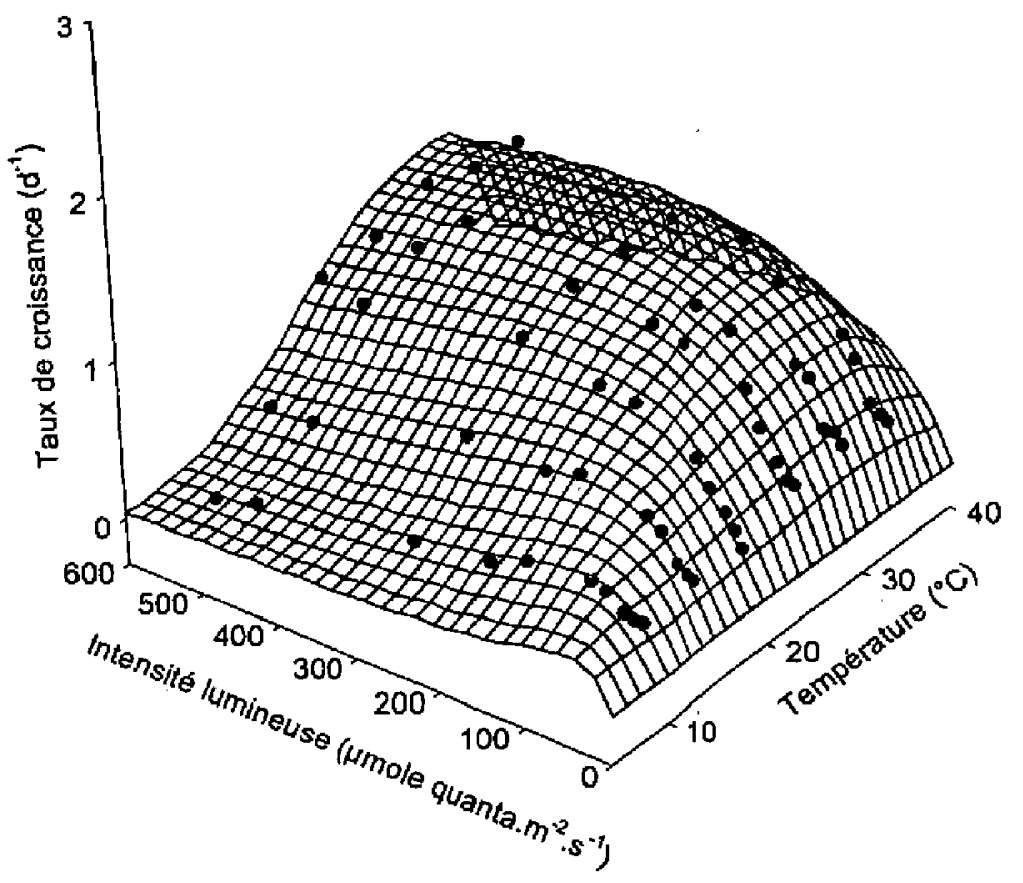

Fig. 2. Interaction de l'intensité lumineuse et de la température sur le taux de croissance de Scenedesmus dimorphus.

Fig. 2. Interaction between light intensity and temperature on the growth rate of Scenedesmus dimorphus.

Les valeurs des paramètres de ce modèle sont reportées dans le tableau 1. Cet ensemble de données montre qu'à chaque température peuvent être définis un taux de croissance maximal $\left(\mu \max _{T}\right)$ et une intensité lumineuse optimale associée ( Iopt $\left._{\mathrm{T}}\right)$. L'évolution de ces deux variables en fonction de la température (Figs. 4,5 ) peut être ajustée à l'aide du modèle de Lehman et al. (1975). Ce modèle (équations 2 \& 2'), restitue de

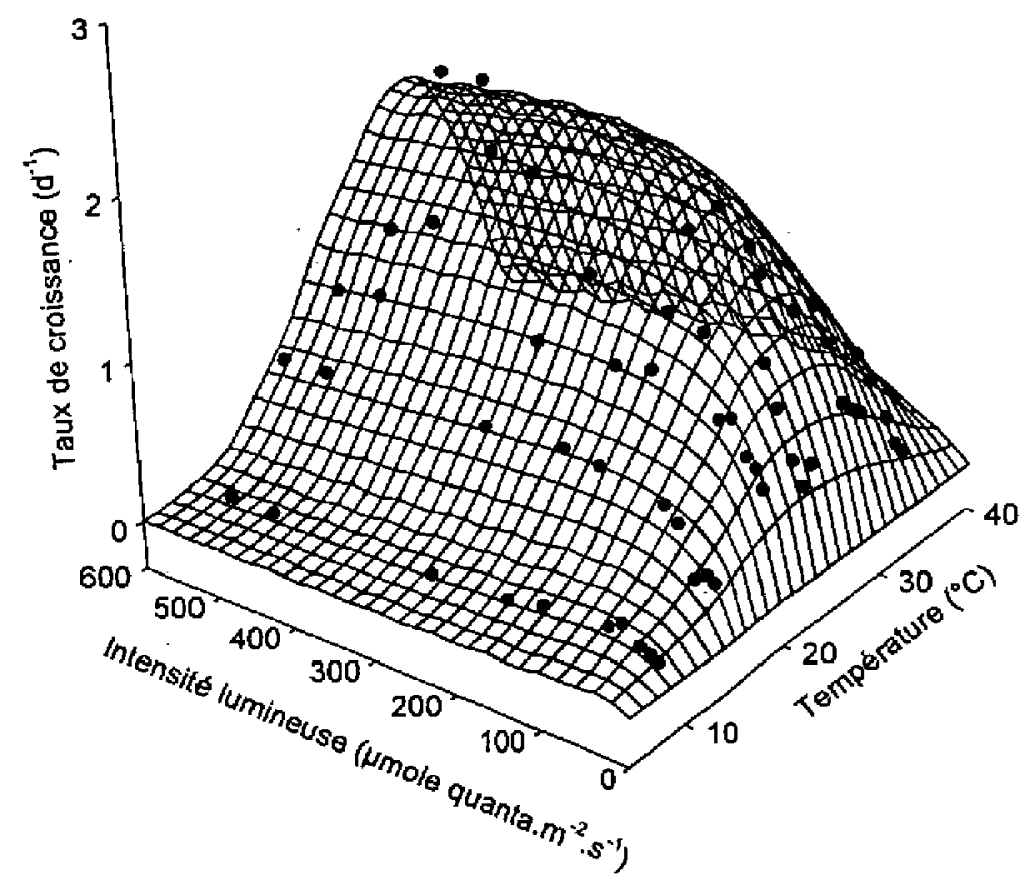

Fig. 3. Interaction de l'intensité lumineuse et de la température sur le taux de croissance de Scenedesmus ecormis.

Fig. 3. Interaction between light intensity and temperature on the growth rate of Scenedesmus ecornis. 


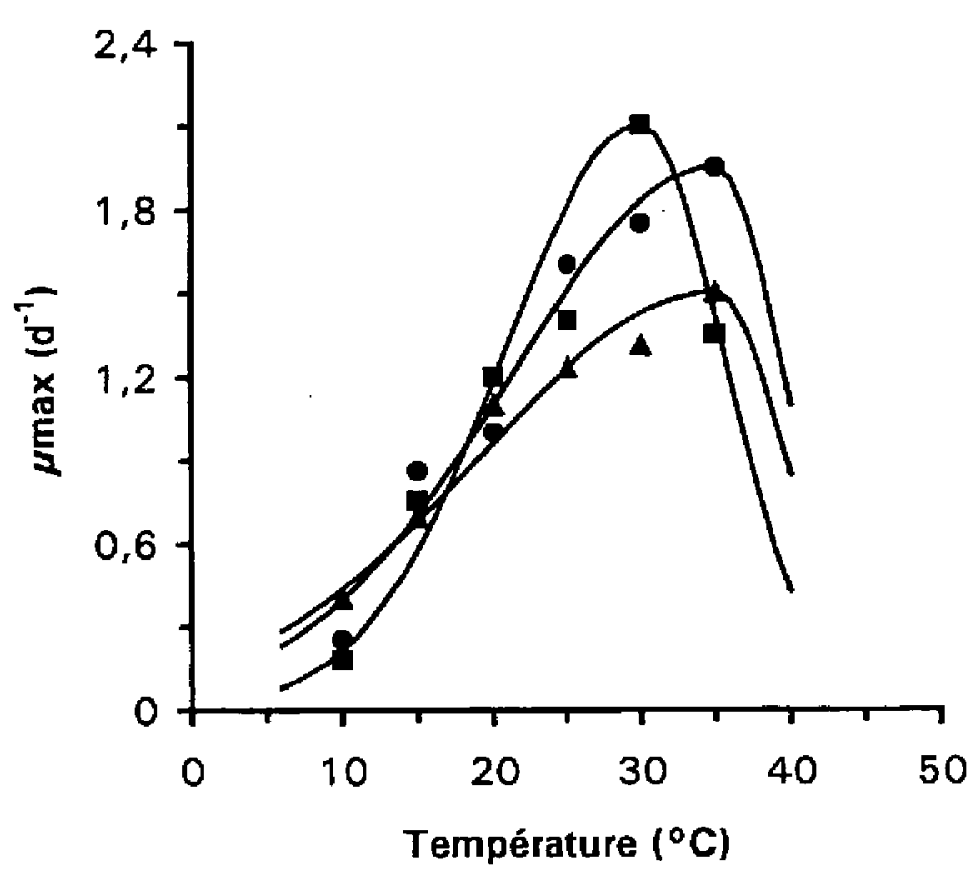

Fig. 4. Evolution du taux de croissance maximal $\left(\mu \max _{\mathrm{T}}\right)$ de $S$.

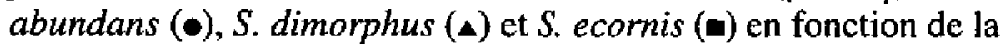
température.

Fig. 4. Maximum growth rate $\left(\mu_{\max }\right)$ of $S$. abundans $(\bullet)$, S. dimorphus (A) and S. ecornis (a) in relation to temperature.

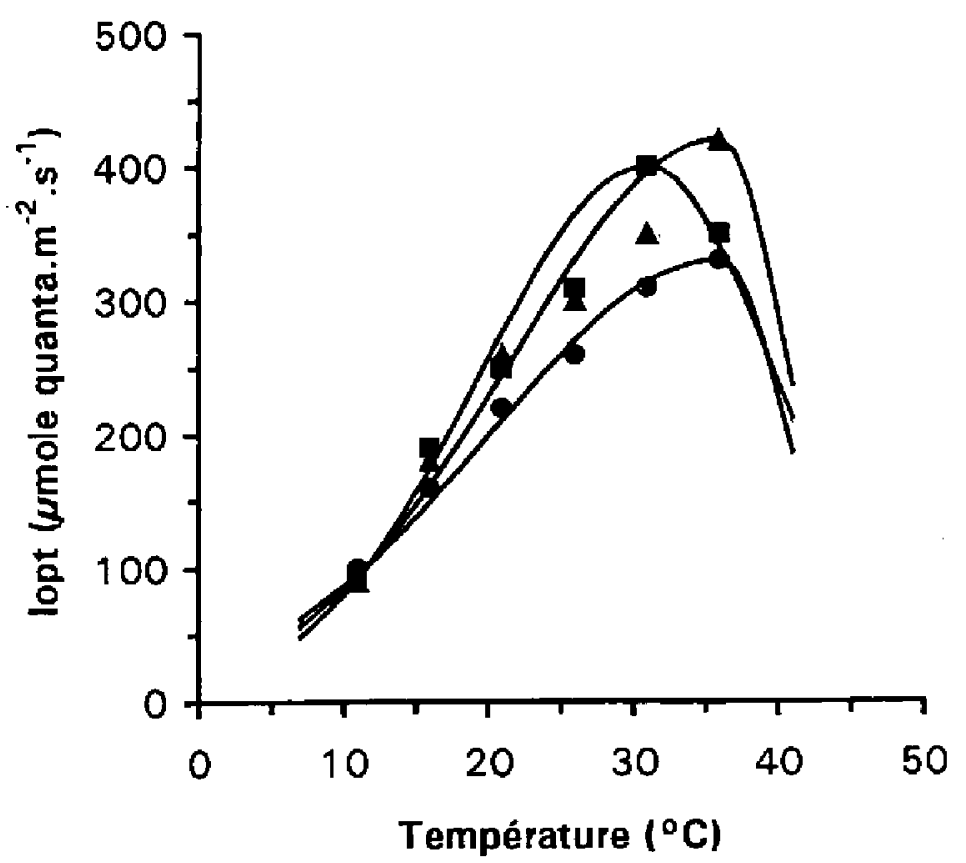

Fig. 5. Evolution de l'intensité lumineuse optimale de croissance

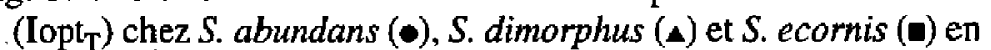
fonction de la température.

Fig. 5. Optimal light intensity (Iopt $)$ of $S$. abundans (๑), S. dimorphus (A) and S. ecornis (a) in relation to temperature.

Tableau 1. Scenedesmus spp. : valeurs des paramètres $\mu$ max, Iopt et $\beta$ dérivés de l'équation de Peeters \& Eilers (1978), en fonction de la température.

Table 1. Scenedesmus spp. : values of parameters $\mu$ max, Iopt and $\dot{\beta}$ derived from Peeters \& Eilers's (1978) equation, in relation to temperature.

\begin{tabular}{|c|c|c|c|c|c|c|c|}
\hline \multirow{2}{*}{ Espèce } & & \multicolumn{6}{|c|}{ Température $\left({ }^{\circ} \mathrm{C}\right)$} \\
\hline & & 10 & 15 & 20 & 25 & 30 & 35 \\
\hline \multirow{3}{*}{ S. abundans } & $\mu \max \left(d^{-1}\right)$ & 0,25 & 0,86 & 1,00 & 1,60 & 1,75 & 1,95 \\
\hline & Iopt ( $\mu$ mole quanta. $\left.\mathrm{m}^{-2} \cdot \mathrm{s}^{-1}\right)$ & 100 & 160 & 220 & 260 & 310 & 330 \\
\hline & $\beta$ & 0,6 & 1,3 & 0,9 & 2,5 & 1,2 & 1,0 \\
\hline \multirow{3}{*}{ S. dimorphus } & $\mu \max \left(d^{-1}\right)$ & 0,40 & 0,69 & 1,10 & $\cdot 1,23$ & 1,31 & 1,50 \\
\hline & Iopt ( $\mu$ mole quanta. $\left.\mathrm{m}^{-2} \cdot \mathrm{s}^{-1}\right)$ & 90 & 180 & 260 & 300 & 350 & 420 \\
\hline & $\beta$ & 0 & 0,2 & 0,3 & 1 & 1,3 & 1,2 \\
\hline \multirow{3}{*}{ S. ecornis } & $\mu \max \left(d^{-1}\right)$ & 0,18 & 0,75 & 1,20 & 1,40 & 2,10 & 1,35 \\
\hline & Iopt $\left(\mu\right.$ mole quanta. $\left.\mathrm{m}^{-2} \cdot \mathrm{s}^{-1}\right)$ & 90 & 190 & 250 & 310 & 400 & 350 \\
\hline & $\beta$ & 0,1 & 1,3 & 2 & 1 & 0,7 & 0,5 \\
\hline
\end{tabular}


manière assez fidèle, les variations de $\mu \max _{\mathrm{T}}$ et de Iopt $_{T}$ lorsque la température de culture s'écarte d'une valeur optimale (Topt) caractéristique de chaque espèce.

$$
\begin{aligned}
& \mu \max _{\mathrm{T}}=\mu \max . \exp \left[-2,3 .(\mathrm{T}-\mathrm{Topt})^{2} / \mathrm{B}^{2}\right] \\
& \text { Iopt }_{\mathrm{T}}=\text { Iopt . } \exp \left[-2,3 .(\mathrm{T}-\mathrm{Topt})^{2} / \mathrm{B}^{2}\right] \\
& \text { avec } \quad B=\text { Tsup - Topt si } \mathrm{T}>\text { Topt } \\
& \mathrm{B}=\text { Tinf - Topt } \text { si } \mathrm{T}<\mathrm{Topt}
\end{aligned}
$$

Tinf et Tsup correspondent respectivement aux températures inférieure et supérieure pour lesquelles $\mu \max _{\mathrm{T}}=0,1 . \mu \max$, et $\operatorname{lopt}_{\mathrm{T}}=$ 0,1 . Iopt

Les valeurs des paramètres des deux sous-modèles sont regroupées dans le tableau 2. L'optimum thermique (Figs. 4,5$)$ pour les deux variables $\left(\mu \max _{T}\right.$ et Iopt $_{\mathrm{T}}$ ) se situe à $30^{\circ} \mathrm{C}$ dans le cas de $S$. ecornis et à $35^{\circ} \mathrm{C}$ pour les deux autres espèces. Entre 10 et $20^{\circ} \mathrm{C}$, l'influence de la température sur la croissance est nettement plus accentuée chez $S$. ecornis et $S$. abundans, comme en témoignent les valeurs de $\mathrm{Q}_{10}$ calculées dans cet intervalle (Tableau 3). Entre 20 et $30^{\circ} \mathrm{C}$, l'effet de la température devient relativement moins important, avec une baisse très sensible des valeurs de $\mathrm{Q}_{10}$ (Tableau 3). D'une manière générale, les $\mathrm{Q}_{10}$ moyens, déterminés selon la méthode décrite par Goldman \& Carpenter (1974), indiquent un comportement similaire, vis-à-vis de la température, chez $S$. ecornis et $S$. abundans par comparaison à $S$. dimorphus. En terme de vitesse de croissance, $S$. ecornis se caractérise par le taux maximal ( $\mu$ max) le plus élevé $\left(2,1 \mathrm{~d}^{-1}\right)$, suivi de $S$. abundans $\left(1,95 \mathrm{~d}^{-1}\right)$ puis de $S$. dimorphus (1,5 $\mathrm{d}^{-1}$ ) (Fig. 4). Ces taux de croissance sont atteints sous des éclairements relativement importants compris entre 330 et $420 \mu$ mole quanta. $\mathrm{m}^{-2} \mathrm{~s}^{-1}$, selon l'espèce considérée (Fig. 5). Par ailleurs, la mise en évidence, pour chacune des trois espèces, d'une forte
Tableau 2. Scenedesmus spp. : valeurs des paramètres des fonctions $\mu \max _{\mathrm{T}}=\mathrm{f}(\mathrm{T})$ et $\mathrm{Iopt}_{\mathrm{T}}=\mathrm{f}(\mathrm{T})$ dérivés de l'équation de Lehman et al. (1975).

Table 2. Scenedesmus spp. : parameters values of $\mu \max _{\mathrm{T}}=\mathrm{f}(\mathrm{T})$ and Iopt $_{\mathrm{T}}=\mathrm{f}(\mathrm{T})$ functions, derived from equation of Lehman et al. (1975).

\begin{tabular}{llccc}
\hline \multirow{2}{*}{ Espèce } & & \multicolumn{3}{c}{ Température $\left({ }^{\circ} \mathrm{C}\right)$} \\
\cline { 3 - 5 } & & Tinf & Topt & Tsup \\
\hline \multirow{2}{*}{ S. abundans } & $\mu \max _{\mathrm{T}}=\mathrm{f}(\mathrm{T})$ & 5 & 35 & 45 \\
& $\mathrm{Iopt}_{\mathrm{T}}=\mathrm{f}(\mathrm{T})$ & 1 & 35 & 45 \\
\hline \multirow{2}{*}{ S. dimorphus } & $\mu \max _{\mathrm{T}}=\mathrm{f}(\mathrm{T})$ & 1 & 35 & 45 \\
& $\operatorname{Iopt}_{\mathrm{T}}=\mathrm{f}(\mathrm{T})$ & 4 & 35 & 45 \\
\hline \multirow{2}{*}{ S. ecornis } & $\mu \max _{\mathrm{T}}=\mathrm{f}(\mathrm{T})$ & 10 & 30 & 42 \\
& $\operatorname{Iopt}_{\mathrm{T}}=\mathrm{f}(\mathrm{T})$ & 5 & 30 & 49 \\
\hline
\end{tabular}

corrélation positive entre $\operatorname{Iopt}_{\mathrm{T}}$ et $\mu \max _{\mathrm{T}}$ révèle l'existence d'une interaction étroite entre la température et l'intensité lumineuse sur les taux de croissance de ces microalgues (Fig. 6).

Parallèlement à l'influence de l'intensité lumineuse, l'effet de la durée d'éclairement (photopériode) sur la vitesse de croissance des trois algues a également été recherché. Les mesures effectuées montrent en effet que le taux de croissance de chaque espèce s'accroît de manière importante avec la durée de l'héméropériode (Fig. 7), atteignant la valeur maximale sous éclairement continu ( $S$. ecornis : $2,47 \mathrm{~d}^{-1} ; S$. abundans : 2,08 $\mathrm{d}^{-1} ; S$. dimorphus $\left.: 1,56 \mathrm{~d}^{-1}\right)$. L'allure générale des résultats obtenus pour les trois espèces a permis de choisir la fonction mathématique décrite par Belkoura \&

Tableau 3. Valeurs de $\mathrm{Q}_{10}$ des trois espèces de Scenedesmus.

Table 3. $\mathrm{Q}_{10}$ values of the three Scenedesmus species.

\begin{tabular}{cccc}
\hline & \multicolumn{3}{c}{ Espèce } \\
\cline { 2 - 4 } Intervalle de température & S. abundans & S. dimorphus & S. ecornis \\
\hline $10^{\circ} \mathrm{C}-20^{\circ} \mathrm{C}$ & $4,00^{\mathrm{a}}$ & $2,75^{\mathrm{a}}$ & $6,67^{\mathrm{a}}$ \\
$20^{\circ} \mathrm{C}-30^{\circ} \mathrm{C}$ & $1,75^{\mathrm{a}}$ & $1,19^{\mathrm{a}}$ & $1,75^{\mathrm{a}}$ \\
$10^{\circ} \mathrm{C}-35^{\circ} \mathrm{C}$ & $2,17^{\mathrm{b}}$ & $1,65^{\mathrm{b}}$ & $2,10^{\mathrm{b}}$ \\
\hline
\end{tabular}

\footnotetext{
a valeurs calculées à partir des données de $\mu$ max figurant dans le tableau 1.

b : valeurs déterminées à partir de la représentation en coordonnées semi-logarithmiques de l'évolution de $\mu$ max en fonction de $1 / \mathrm{T}$ (avec $\mathrm{T}$ en ${ }^{\circ} \mathrm{Kelvin}$ ), selon la méthode décrite par Goldman \& Carpenter (1974).
} 


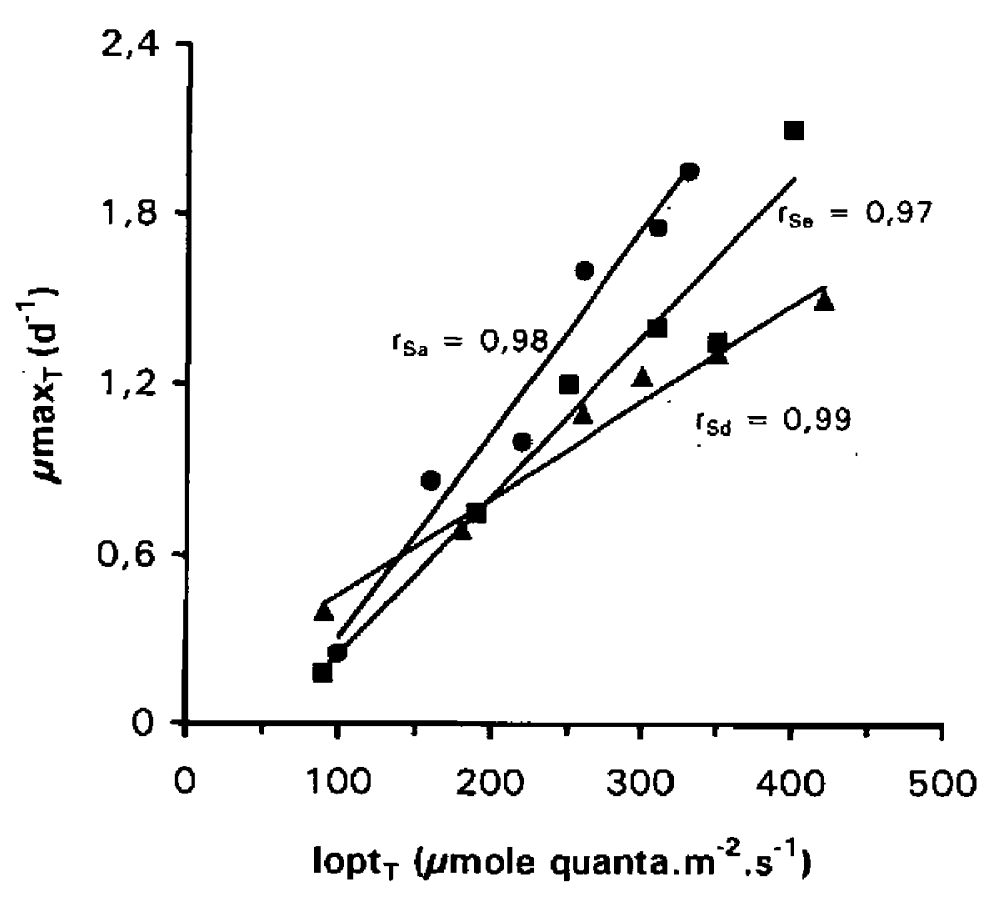

Fig. 6. Corrélation entre le taux de croissance maximal $\left(\mu \max _{\mathrm{T}}\right)$ et l'intensité lumineuse optimale (lopt $\mathrm{T}_{\mathrm{T}}$ ) chez $S$. abundans $(\bullet, \mathrm{Sa})$, $S$. dimorphus (^, Sd) et $S$. ecornis ( $\mathbf{\sim}, \mathrm{Se})$.

Fig. 6. Correlation between maximum growth rate $\left(\mu \max _{\mathrm{T}}\right)$ and optimal light intensity ( $\mathrm{Iopt}_{\mathrm{T}}$ ) of $S$. abundans $(\bullet, \mathrm{Sa}), S$. dimorphus $(\Lambda, \mathrm{Sd})$ and $S$. ecomis $(\boldsymbol{\Pi}, \mathrm{Se})$.

Dauta (1992) pour modéliser l'évolution du taux de croissance en fonction de la photopériode :

$\mu \max _{(30, n H)}=\mu \max _{(30,15 / 9)} \cdot\left[A+1 /\left(B+C \cdot e^{(D-E . n H)}\right)\right]$

Cette équation peut être extrapolée pour toutes les combinaisons Température-Photopériode $(\mathrm{T}, \mathrm{nH})$ par la relation suivante :

$\mu \max _{(\mathrm{T}, \mathrm{nH})}=\mu \max _{(\mathrm{T}, 15 / 9)} \cdot\left[\mathrm{A}+1 /\left(\mathrm{B}+\mathrm{C} . \mathrm{e}^{(\mathrm{D}-\mathrm{E} \cdot \mathrm{nH})}\right)\right](4)$

Le tableau 4 regroupe les coefficients (A, B, C, D et E) spécifiques à chaque espèce.

Tableau 4. Scenedesmus spp. : valeurs des coefficients de l'équation de Belkoura \& Dauta (1992).

Table 4. Scenedesmus spp. : coefficients values of Belkoura \& Dauta's (1992) aquation.

\begin{tabular}{lccccc}
\hline Espèce & $\mathrm{A}$ & $\mathrm{B}$ & $\mathrm{C}$ & $\mathrm{D}$ & $\mathrm{E}$ \\
\hline S. abundans & $-2,15$ & 0,287 & 0,85 & $-1,54$ & 0,12 \\
S. dimorphus & $-2,28$ & 0,275 & 0,75 & $-1,49$ & 0,10 \\
S. ecornis & $-2,27$ & 0,275 & 0,94 & $-1,68$ & 0,11 \\
\hline
\end{tabular}

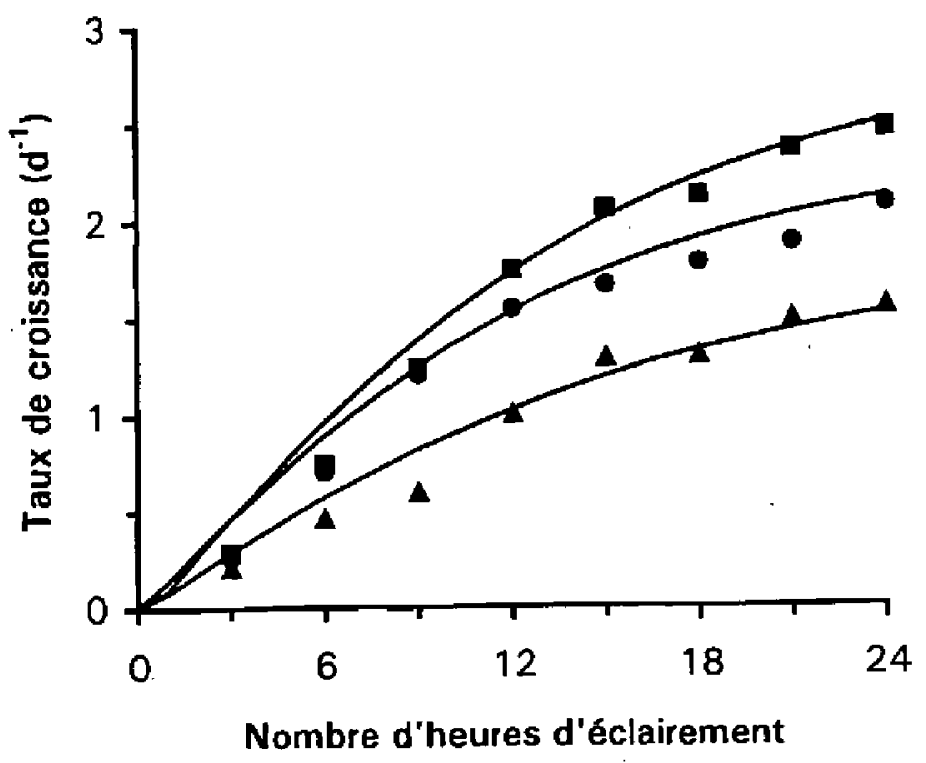

Fig. 7. Evolution du taux de croissance de $S$. abundans (๑), $S$. dimorphus ( $\Delta$ ) et $S$. ecornis ( $\boldsymbol{(})$ en fonction du nombre d'heures d'éclairement.

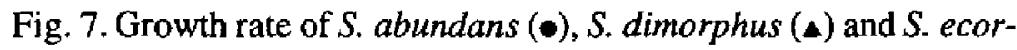
nis (a) vs. daylength.

\section{Discussion}

A côté des espèces du genre Chlorella, Dunaliella, ou encore Chlamydomonas, les microalgues du genre Scenedesmus font partie actuellement des chlorophycées les plus exploitées à l'échelle industrielle, en raison notamment de leur productivité élevée. Les différentes études réalisées en laboratoire ont montré en effet que les espèces du genre Scenedesmus se caractérisaient par une croissance rapide, avec des taux compris entre 1,5 et $2 \mathrm{~d}^{-1}$ (Tableau 5). Les faibles taux de croissance ( $<$ à $\left.1,5 \mathrm{~d}^{-1}\right)$ rapportés par certains auteurs (Mouget et al. 1995, Nicklisch 1998) sont probablement en relation avec des conditions de culture non optimales. Dans le cas des trois espèces étudiées, les taux de croissance maximaux enregistrés s'insèrent dans l'intervalle cité précédemment (Tableau 5), avec une variabilité interspécifique. Il faut noter cependant que les données de productivité ou les taux de croissance mesurés en laboratoire ne reflètent pas toujours les valeurs réelles de rendements dans les systèmes de culture en masse. En conditions naturelles, les variations journalières et saisonnières des conditions environnementales, associées parfois à des problèmes d'ordre technique ou biologique (pannes techniques, infections des cultures par des parasites ou des prédateurs), ont un impact sur le rendement de ces systèmes. Ainsi, la connaissance de l'influence des facteurs héliother- 
miques (température, lumière) sur la croissance des microalgues et la modélisation de ces processus permettent, à priori, de mieux cerner les capacités de chaque espèce à se développer dans des unités de production sous les conditions naturelles.

L'étude réalisée sur $S$. abundans, $S$. dimorphus et $S$. ecornis a permis de mettre en évidence un accroissement particulièrement important du taux de croissance des trois espèces avec la température; l'optimum thermique se situant à $30^{\circ} \mathrm{C}$ pour $S$. ecormis et à $35^{\circ} \mathrm{C}$ pour les deux autres microalgues. Selon Richmond (1986b), ces trois espèces pourraient donc être considérées comme des algues mésophiles. Dans leur revue synthétique du genre Scenedesmus, Soeder \& Hegewald (1988) rapportent des températures optimales de croissance comprises, toutes espèces confondues, entre 30 et $36^{\circ} \mathrm{C}$. Cependant, Hsia et al. (1975) ont signalé l'existence d'une souche thermophile de $S$. obliquus HB 6105 possédant un optimum thermique à $42^{\circ} \mathrm{C}$. De même, Hegewald (1984) a pu isoler, à partir de différents écosystèmes aquatiques des régions tropicales, des souches de $S$. communis et de $S$. perforatus ayant une croissance optimale à des températures comprises entre 38 et $42^{\circ} \mathrm{C}$. Par ailleurs, au-delà de cette notion de température optimale, les expériences réalisées ont révélé que les trois espèces étudiées pouvaient se développer à des températures nettement plus basses ( 10 et $15^{\circ} \mathrm{C}$ notamment). Cette dernière caractéristique indique que ces microalgues sont capables de supporter des fluctuations thermiques pouvant atteindre $20^{\circ} \mathrm{C}$. Selon Becker (1994), ce type de comportement vis-à- vis de la température est un des critères de sélection des espèces destinées à la culture en masse, en particulier dans des régions où les amplitudes thermiques journalières peuvent atteindre des valeurs aussi importantes.

Associée à la température, la lumière est la variable majeure agissant sur la croissance des microalgues, avec deux paramètres : l'intensité de l'éclairement et la durée de l'héméropériode. Les résultats de cette étude ont montré que les trois espèces de Scenedesmus se caractérisaient par une intensité lumineuse optimale de croissance sensiblement plus élevée (entre 330 et $420 \mu$ mole quanta. $\mathrm{m}^{-2} \cdot \mathrm{s}^{-1}$ ) par rapport à d'autres microalgues du même genre : de 250 à $300 \mu$ mole quanta. $\mathrm{m}^{-2} \cdot \mathrm{s}^{-1}$ pour $S$. crassus (Guerri et al. 1981, Dauta 1982, Derraz et al. 1995), $300 \mu$ mole quanta. $\mathrm{m}^{-2} \cdot \mathrm{s}^{-1}$ pour $S$. quadricauda (Dauta 1982), $350 \mu$ mole quanta. $\mathrm{m}^{-2} . \mathrm{s}^{-1}$ dans le cas de $S$. bicellularis (Mouget et al. 1995). Cette relative variabilité interspécifique peut s'expliquer notamment par l'existence d'une interaction étroite entre l'intensité lumineuse optimale de croissance d'une part et la température d'autre part. En effet, plus les températures optimales de croissance sont élevées plus les besoins en lumière deviennent importants pour saturer l'activité photosynthétique de ces organismes autotrophes (Derraz et al. 1995). Dans le cas des trois microalgues étudiées, la mise en évidence d'une forte corrélation positive entre Iopt ${ }_{\mathrm{T}}$ et $\mu$ max confirme cette hypothèse. D'une manière générale, les nombreuses études réalisées sur les chlorophycées ont montré que ces algues possédaient des op-

Tableau 5. Données de la littérature relatives aux taux de croissance de diverses espèces du genre Scenedesmus. Table 5. Literature data for the growth rates in different Scenedesmus species.

\begin{tabular}{|c|c|c|c|}
\hline Espèce & $\mu \max$ & Conditions de culture & Référence \\
\hline S. abundans & $1,95 \mathrm{~d}^{-1}$ & $35^{\circ} \mathrm{C}, 330 \mu$ mole quanta. $\mathrm{m}^{-2} \mathrm{~s}^{-1}, 15 / 9 \mathrm{~L} / \mathrm{O}$ & Présent travail \\
\hline S. acuminatus & $0,81 \mathrm{~d}^{-1}$ & $20^{\circ} \mathrm{C}, 190$ umole quanta. $\mathrm{m}^{-2} \mathrm{~s}^{-1}, 12 / 12 \mathrm{~L} / \mathrm{O}$ & Nicklisch 1998 \\
\hline S. armatus & $1,10 \mathrm{~d}^{-1}$ & $20^{\circ} \mathrm{C}, 190 \mu$ mole quanta $\mathrm{m}^{-2} \mathrm{~s}^{-1}, 12 / 12 \mathrm{~L} / \mathrm{O}$ & Nicklisch 1998 \\
\hline S. bicellularis & $1,27 \mathrm{~d}^{-1}$ & $23^{\circ} \mathrm{C}, 350 \mu$ mole quanta. $\mathrm{m}^{-2} \mathrm{~s}^{-1}, 12 / 12 \mathrm{~L} / \mathrm{O}$ & Mouget et al. 1995 \\
\hline S. crassus & $1,62 \mathrm{~d}^{-1}$ & $30^{\circ} \mathrm{C}, 250$ umole quanta. $\mathrm{m}^{-2} \mathrm{~s}^{-1}, 15 / 9 \mathrm{~L} / \mathrm{O}$ & Dauta 1982 \\
\hline S. crassus & $1,72 \mathrm{~d}^{-1}$ & $30^{\circ} \mathrm{C}, 300$ umole quanta $\mathrm{m}^{-2} \mathrm{~s}^{-1}, 15 / 9 \mathrm{~L} / \mathrm{O}$ & Derraz et al. 1995 \\
\hline S. crassus & $1,60 \mathrm{~d}^{-1}$ & $32^{\circ} \mathrm{C}, 260$ umole quanta. $\mathrm{m}^{-2} \mathrm{~s}^{-1}, 15 / 9 \mathrm{~L} / \mathrm{O}$ & Guerri et al. 1981 \\
\hline S. dimorphus & $1,50 \mathrm{~d}^{-1}$ & $35^{\circ} \mathrm{C}, 420 \mu$ mole quanta $\mathrm{m}^{-2} \mathrm{~s}^{-1}, 15 / 9 \mathrm{~L} / \mathrm{O}$ & Présent travail \\
\hline S. ecornis & $2,10 \mathrm{~d}^{-2}$ & $30^{\circ} \mathrm{C}, 400 \mu$ mole quanta. $\mathrm{m}^{-2} \mathrm{~s}^{-1}, 15 / 9 \mathrm{~L} / \mathrm{O}$ & Présent travail \\
\hline S. obliquus & $1,52 \mathrm{~d}^{-1}$ & $25^{\circ} \mathrm{C}, 98$ umole quanta $\mathrm{m}^{-2} \mathrm{~s}^{-1}$ & Sorokin \& Krauss 1958 \\
\hline S. protuberans & $1,58 \mathrm{~d}^{-1}$ & $20^{\circ} \mathrm{C}, 160 \mu$ mole quanta. $\mathrm{m}^{-2} \mathrm{~s}^{-1}$ & Mur et al. 1978 \\
\hline S. quadricauda & $1,71 \mathrm{~d}^{-1}$ & $32^{\circ} \mathrm{C}, 300 \mu$ mole quanta $\mathrm{m}^{-2} \mathrm{~s}^{-1}, 15 / 9 \mathrm{~L} / \mathrm{O}$ & Dauta 1982 \\
\hline S. quadricauda & $1,79 \mathrm{~d}^{-1}$ & $20^{\circ} \mathrm{C}, 24 / 0 \mathrm{~L} / 0$ & Oh \& Rhee 1991 \\
\hline
\end{tabular}


timums d'éclairement nettement plus importants par rapport à d'autres groupes tels que les cyanophycées ou les diatomées (Mur et al. 1978, Dauta 1982, Reynolds 1984, Boumnich et al. 1990, Dauta et al. 1990, Nicklisch 1998). Par ailleurs, outre l'influence directe de l'intensité lumineuse sur la croissance, la photopériode joue également un rôle important sur la croissance des microalgues à travers notamment son impact sur divers processus cellulaires (rythmes circadiens de la photosynthèse et de la respiration, divisions, activités enzymatiques, etc...). Les expériences réalisées ont révélé en effet une nette augmentation du taux de croissance des trois espèces de Scenedesmus en fonction de la durée de l'héméropériode, avec des valeurs maximales sous éclairement continu. Ce résultat est en accord avec de nombreux travaux réalisés sur diverses espèces d'algues (Foy et al. 1976, Dauta 1982, Sakshaug \& Andresen 1986, Belkoura \& Dauta 1992, Nielsen 1992, Foy \& Gibson 1993). Cependant, la prise en compte du rapport entre le coût énergétique et la production de biomasse correspondante montre que des durées d'éclairement de 12 à 15 heures sont les plus adéquates. Ainsi, l'ensemble des données expérimentales indique que les trois espèces de Scenedesmus étudiées sont parfaitement adaptées aux conditions d'ensoleillement (intensité et photopériode) enregistrées dans des régions à climat semi-aride, voire même aride.

La modélisation des cultures offre la possibilité de prédire l'évolution d'une population d'algues en fonction des facteurs environnementaux. Diverses formulations ont été employées par de nombreux auteurs pour traduire, en terme d'équations mathématiques simples, l'influence des paramètres de culture (intensité lumineuse, température, photopériode, nutriments, etc...) sur la croissance des microalgues (Ten Hoopen et al. 1981, Feuillade \& Feuillade 1987, Cornet et al. 1992a,b, Belkoura 1994). Parmi les nombreuseses équätions disponibles dans la littérature, celles de Peeters \& Eilers (1978), de Lehman et al. (1975) et de Belkoura \& Dauta (1992) ont permis de restituer, de manière très satisfaisante, l'influence de l'intensité lumineuse, de la température et de la photopériode sur la croissance des trois espèces de Scenedesmus étudiées. La mise en évidence d'une forte corrélation positive entre les taux de croissance mesurés au cours d'expériences diverses ( $\mu$ obs) et ceux prédits ( $\mu \mathrm{mod})$ par les différentes équations retenues démontre d'ailleurs la validité de ces modèles dans le cas des trois microalgues (Fig. 8). Ainsi, il devient donc possible de prédire le taux de croissance de l'une ou de l'autre des trois espèces pour toute combinaison d'éclairement (intensité, photopé-
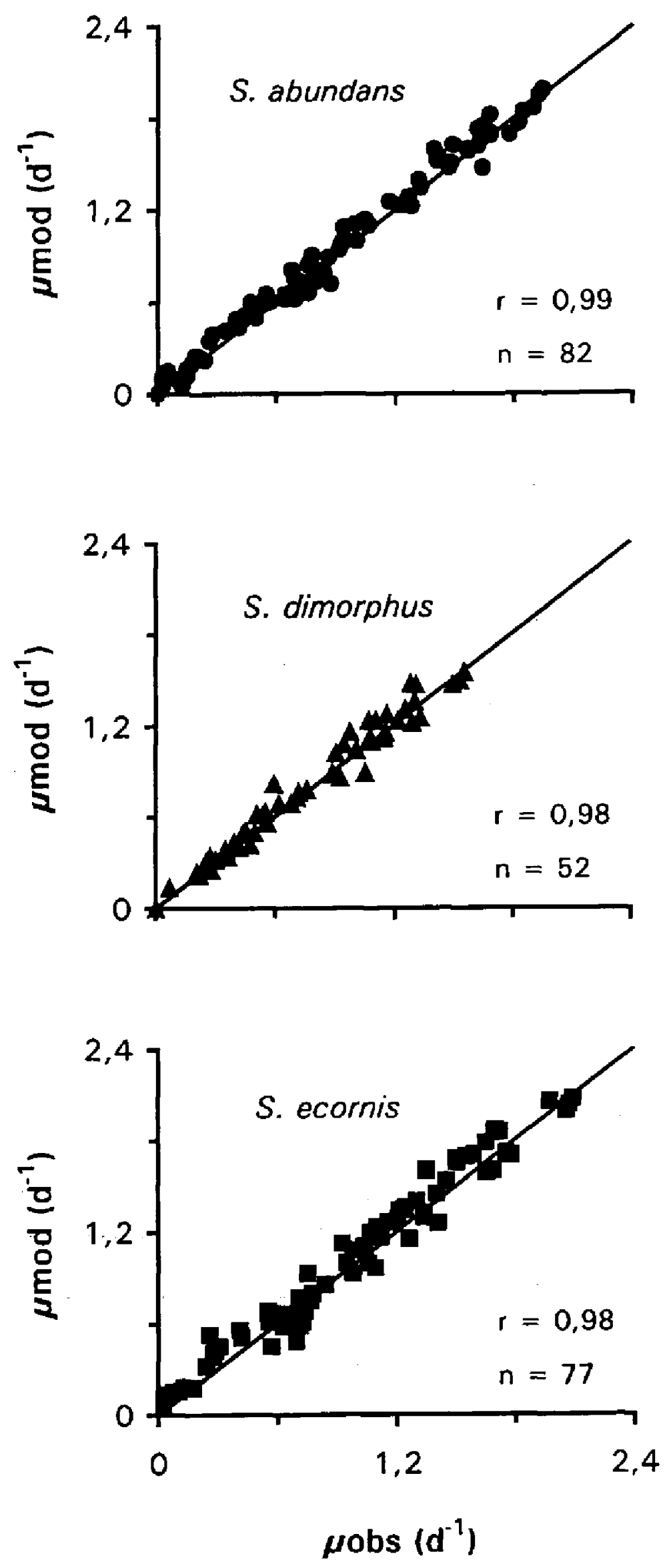

Fig. 8. Comélation entre les taux de croissance observés ( $\mu$ obs) et les taux de croissance modélisés ( $\mu \mathrm{mod})$ dans le cas des 3 espèces de Scenedesmus.

Fig. 8. Correlation between the growth rates observed ( $\mu$ obs) and the growth rates expected ( $\mu$ mod) by the used models, for the 3 Scenedesmus species. 
riode) et de température. Ce set de données, associé aux différentes formulations mathématiques, fournit ainsi la base d'un modèle global de simulation de cultures d'algues. Afin de disposer d'un outil performant utile pour I'étude préliminaire de systèmes de culture d'algues à vocation appliquée, ce modèle devra prendre en compte également des processus fondamentaux tels que l'assimilation et le stockage des nutriments, l'agitation et les effets de l'auto-ombrage.

\section{Remerciements}

Ce travail a été financé conjointement par l'Agence Universitaire de la Francophonie (ex AUPELF-UREF), dans le cadre d'un contrat d'association (JER 5001) du Fond Francophone de la Recherche, et par le Ministère Marocain de l'Enseignement Supérieur, de la Formation des Cadres et de la Recherche Scientifique, dans le cadre d'un contrat PROTARS (P1T1/36).

La souche de $S$. dimorphus nous a été aimablement fournie par Abdelkhalek Toumi que nous remercions vivement. Les auteurs remercient également le Professeur Mohamed Loudiki, Responsable du Laboratoire d'Algologie de la Faculté des Sciences Semlalia, pour l'identification des espèces d'algues utilisées dans cette étude.

\section{Travaux cités}

Becker E.W. 1994. - Microalgae : Biotechnology and Microbiology. Baddiley J., Carey N.H., Higgins 1.J. \& Potter W.G. (Eds), Cambridge Studies in Biotechnology, Cambridge : 293 p.

Becker E.W. \& Venkatamaran L.V. 1980. - Production and processing of algae in pilot plant scale experiences of the Indo-German Project. In Algae Biomass. Shelef G. \& Soeder C.J., (Eds.), Elsevier Press, Amsterdam: 35-50.

Becker E.W. \& Venkataraman L.V. 1982. - Biotechnology and exploitation of algae : the Indian approach. Eschborn: German Agency for Technical Cooperation GmbH.

Belkoura M. 1994. - Chlorella sorokininana Shihira \& Krauss : ecophysiologie, modélisation de cultures et perspectives de valorisation de la biomasse algale. Thèse de Doctorat d'État esSciences, Université Cadi Ayyad, Faculté des Sciences Semlalia, 154 p. (tannexe).

Belkoura M. \& Dauta A. 1992. - Interaction lumière-température et influence de la photopériode sur le taux de croissance de Chlorella sorokiniana Shihira \& Krauss. Ann. Limnol., 28(2) : 101107.

Benider A. 2001. - Perspectives de valorisation de quelques microalgues vertes (Chlorophyceae) : optimisation de la production de biomasse, caractérisation biochimique et recherche d'activité antibactérienne. Thèse de Doctorat d'État es-Sciences, Université Cadi Ayyad, Faculté des Sciences Semlalia : 106 p.

Boumnich L., Dauta A., Devaux J. \& Romagoux J.C. 1990. - Influence de la lumière et de la température sur la croissance de quatre espèces d'algues d'un lac eutrophe (Lac d'Aydat, Puy de Dôme, France). Ann. Limnol., 26(1): 3-10.

Castillo J., Merino F. \& Heussler P. 1980. - Production and ecological implications of algae mass culture under Peruvian conditions. In Algae Biomass. Shelef G. \& Soeder C.J., (Eds.), Elsevier Press, Amsterdam : 123-134.

Cornet J.F., Dussap C.G., Cluzel P. \& Dubertret G. 1992a. - A structured model for simulation of cultures of the cyanobacterium Spirulina platensis in photobioreactors: II. Identification of kinetic parameters under light and mineral limitations. Biotechnology and Bioengineering, $40: 826-834$.
Cornet J.F., Dussap C.G. \& Dubertret G. 1992b. - A structured model for simulation of cultures of the cyanobacterium Spirulina platensis in photobioreactors: I. Coupling between light transfer and growth kinetics. Biotechnology and Bioengineering, $40: 817$. 825 .

Dauta A. 1982. - Conditions de développement du phytoplancton. Etude comparative de huit espèces en culture. I. Détermination des paramètres de croissance en fonction de la lumière et de la température, Ann. Limnol., 18(3) : 217-262.

Dauta A., Devaux J., Piquemal F. \& Boumnich L. 1990. - Growth rate of four freshwater algae in relation to light and temperature. Hydrobiologia, $207: 221-226$.

Derraz M., Dauta A., Capblancq J. \& Abassi M. 1995. — Influence de la lumière et de la température sur les taux de croissance et de photosynthèse de Scenedesmus crassus Chodat, isolée de la retenue eutrophe El Kansera (Maroc). Ann. Limnol., 31(1) : 65-74.

Dilov C., Georgiev D. \& Bozhkova M. 1985. - Cultivation and application of microalgae in the People's Republic of Bulgaria. Archiv. Hydrobiol. Ergebnisse der Limnologie, 20 : 35-38.

El-Fouly M.M., Mohn F.H. \& Soeder C.J. 1985. — Joint EgyptianGerman project. Intensive protein production through microalgae. Kernforschungsanlage Jülich GmbH, Publication 34/85.1.

Feuillade J.B. \& Feuillade M. 1987. - Modelling steady-state growth and photosynthesis rates of Oscillatoria rubescens continuous cultures in relation to temperature and irradiance. J. Plankton Res., 9(3) : 445-457.

Foy R.H. \& Gibson C.E. 1993. - The influence of irradiance, photoperiod and temperature on the growth kinetics of three planktonic diatoms. Eur. I. Phycol., 28 : 203-212.

Foy R.H., Gibson C.E. \& Smith R.V. 1976. - The influence of daylength, light intensity and temperature on the growth rates of planktonic blue-green algae. Br. phycol. $J$., $11: 151-163$.

Goldman J.C. \& Carpenter E.J. 1974. - A kinetic approach to the effect of temperature on algal growth. Limnol. Oceanogr., 19(5): 756-766.

Guerri M.M., Brunel L. \& Dauta A. 1981. - Interaction de la lumière et de la température sur le taux de croissance de Scenedesmus crassus. Ann. Limnol., 17(2) : 97-104.

Hegewald E. 1984. - High temperature strains in the genus Scenedesmus, section Desmodesmus. Algological Studies, 37 : 441-446.

Heussler P. 1985. - Aspects of sloped algae pond engineering. Archiv. Hydrobiol. Ergebnisse der Limnologie, 20 : 71-84.

Hsia I.T., Du D.S., Chieng C.H. \& Ley S.H. 1975. — A high-temperature strain of green alga Scenedesmus and its synchronous cell development. Acta Hydrobiol. Sinica, $5: 380-386$.

Lehman J.T., Botkin D.B. \& Likens G.E. 1975. - The assumptions and rationales of a computer model of phytoplankton population dynamics. Limnol. Oceanogr., $20:$ 343-364.

Mouget J.L., de la Noiie J., Legendre L., Jean Y. \& Viarouge P. 1995. - Long-term acclimatization of Scenedesmus bicellularis to high-frequency intermittent lighting $(100 \mathrm{~Hz})$. I. Growth, photosynthesis and photosystem II activity. J. Plankton Res., 17(4) : 859-874.

Mur L.R., Gons H.J. \& Vanlière L. 1978. - Competition of the green alga Scenedesmus and the blue-green algal Oscillatoria. Mitt. Int. Ver. Limnol., $21: 473-479$.

Nicklisch A. 1998. - Growth and light absorption of some planktonic cyanobacteria, diatoms and Chlorophyceae under simulated natural light fluctuations. J. Plankton Res., 20(1) : 105-119.

Nielsen M.V. 1992. - Irradiance and daylength effects on growth and chemical composition of Gyrodinium aureolum Hulburt in culture. J. Plankton Res., 14(6) : 811-820. 
Oh H.M. \& Rhee G.Y. 1991. - A comparative study of microalgae isolated from flooded rice paddlies: light-limited growth, $\mathrm{C}$ fixation, growth efficiency and relative $\mathrm{N}$ and $\mathrm{P}$ requirement. $J$. applied Phycol., $3: 211-220$.

Payer H.D., Pithakpol B., Nguitragool M., Prabharaksa C., Thananunkul D. \& Chavana S. 1978. - Major results of the Thai-German Microalgae Project at Bangkok. Archiv. Hydrobiol. Ergebnisse der Limnologie, $11: 41-55$.

Peeters J.C. \& Eilers P. 1978. - The relationship between light intensity and photosynthesis : a simple mathematical model. $\mathrm{Hy}$ drobiol. Bull., 12 : 134-136.

Reynolds C.S. 1984. - The ecology of freshwater phytoplankton. Beck E., Birks H.J.B. \& Connor E.F. (Eds), Cambridge University Press, London : $384 \mathrm{p}$.

Richmond A. 1986a. - Microalgae of economic potential. In Handbook of Microalgal Mass Culture. Richmond A., (Ed.), CRC Press, Boca Raton, Florida : 285-329.

Richmond A. 1986b. - Cell response to environmental factors. In Handbook of Microalgal Mass Culture. Richmond A., (Ed.), CRC Press, Boca Raton, Florida: 69-99.
Sakshaug E. \& Andresen K. 1986. - Effect of light regime upon growth rate and chemical composition of a clone of Skeletonema costatum from Trondheimsfjord, Norway. J. Plankton Res., 8(4) : 619-637.

Soeder C.J. \& Hegewald E. 1988. - Scenedesmus. In Micro-algal Biotechnology. Borowitzka M.A. \& Borowitzka L.J., (Eds.), Cambridge University Press, London : 59-84.

Sorokin C. \& Krauss R.W. 1958. - The effects of light intensity on the growth rates of green algae. Plant Physiol., 33: 109-113.

Stengel E. \& Soeder C.J. 1975. - Control of photosynthetic production in aquatic ecosystems. In Photosynthesis and Productivity in different Environments. Cooper J.P., (Ed.), Cambridge University Press, Cambridge : 645-660.

Ten Hoopen H.G.J., Roels J.A., Van Gemert J.M., Nobel P.J. \& Fuchs A. 1981. - An unstructured model of algal growth in continuous cultures. In Advances in biotechnology. International Fermentation Symposium. Pergamon, Elmsford, N.Y. : 315-321.

Tahiri M., Benider A., Belkoura M. \& Dauta A. 2000. - Caractérisation biochimique de l'algue verte Scenedesmus abundans : influence des conditions de culture. Ann. Limnol., 36(1) : 3-12. 